\title{
Visual Servo System of Water Washing Robot for Substation
}

\author{
Zhenli Wang ${ }^{1, a}$, Shiyou Mu ${ }^{2, b}$, Jie Ren ${ }^{3, c}$, Shouyin $\mathrm{Lu}^{4, \mathrm{~d}}$, Jian $\mathrm{Li}^{5, \mathrm{e}}$ \\ ${ }^{12345}$ Shandong Electric Power Research Institute, China \\ ${ }^{12345}$ Shandong Luneng Intelligent Co. Ltd \\ ${ }^{12345}$ State Gride Shandong Electric Power Research Institute \\ as sunanger@163.com, 'shiy@Inint.com, 'rj@Inint.com, 'lushy@163.com, ${ }^{\mathrm{e}} \mathrm{lij} @ 163 . c o m$
}

Keywords: Water Washing; Robot; Manipulator; Visual Servo

\begin{abstract}
This paper provides a visual servo system of water washing robot for Substations, which is based on binocular stereo vision algorithms technology. it can ensures the safe operation of the robot and the substation equipments. By utilizing this system to replace the operators, it can semi-automatic wash the equipments in substation for live working so that it can effectively reduce the heavy labor by manual and ensure safety of operators. And also, it can prevent equipments from pollution flash-over accidents happening and guarantee the power grid safe operation.
\end{abstract}

\section{Introduction}

The main reason of flash-over accident in substation equipments is that contaminations deposits on the surface of insulator. Recently, air pollution is increasingly serious, which leads to increase the pollution flashover accidents and seriously affects the safety operation of grid equipments. The way of cleaning contaminations with the electricity cut-off, is not only need to spend huge manpower and material resources, as shown in Fig.1, but also seriously affect the continuity of power supply. So live water washing which does not need to cut off power is widespread used. However, live water washing need high labor intensity, and during the process of live water washing, the distance between operators and equipments is so close that the personal safety of the operator can't be guaranteed.

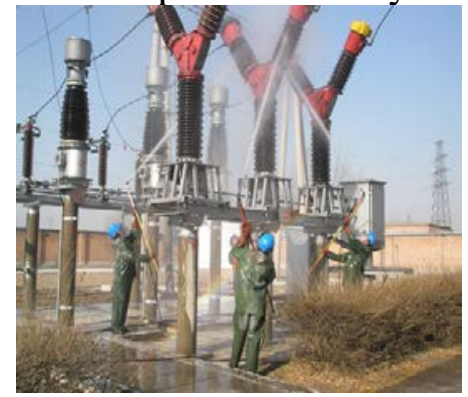

Fig. 1 Washing work by operator

The system of water washing robot for Substations can replace the operators, and it can wash the equipments in substation for live working, so that it effectively reduce the heavy labor of operators and ensure working safety.

\section{Water Washing Robot}

The system of Water washing robot for live working, consists of the robot body subsystem and the high-pressure-pure-water preparation subsystem. The robot body subsystem uses the design of mobile manipulator structure, whose terminal is installed water gun. Trough controlling this subsystem, the robot can complete the complicated live washing work. High-pressure-pure-water preparation subsystem is mainly to achieve the preparation of pure water with high resistivity, boost pressure during the preparation of high pure water, and provide water via a high pressure hose to robot body subsystem. 
The robot body subsystem, as shown in Fig.2, includes hydraulic walk mechanism on the bottom, five-DOF manipulator mounted on the top of walk mechanism, control platform of water gun mounted on the end of manipulator. Big arm rotary joint, big arm pitch joint, small arm telescopic joint, platform pitching joint and platform rotary joint compose the five-DOF manipulator, and all joints use hydraulic dive. Through controlling the opening direction and degree of hydraulic proportion valve by control system, the motion direction and velocity of hydraulic motor can be accurate controlled.

High-pressure-pure-water preparation system, as shown in Fig.3, includes the preparation of highpure-water device, water supply pressure device and other components. The resistivity High pure water produced by the system can reach $1 \mathrm{M} \Omega$; Water supply pressure device uses pressure plunger pump to pressurize prepared pure water, then high pressure hose output transport the pure water to the robot subsystem.

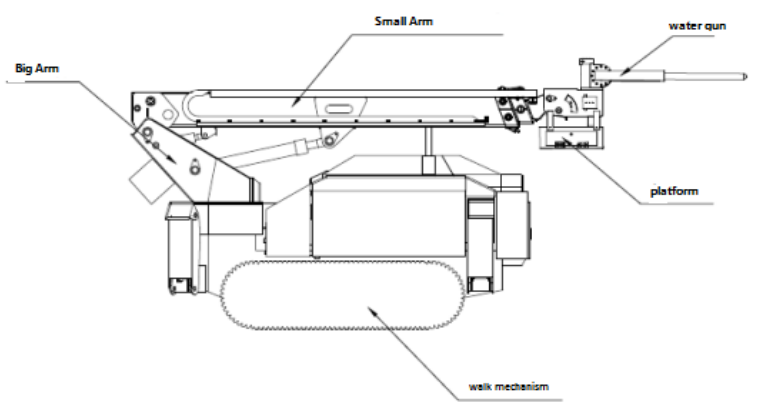

Fig 2 robot body system

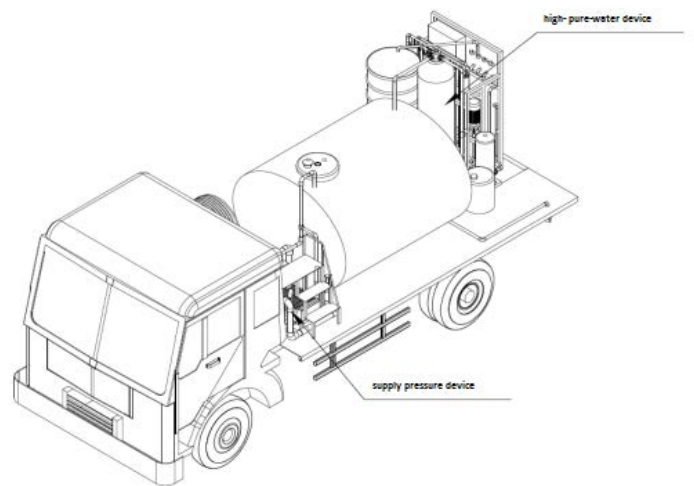

Fig 3 high-pressure-pure-water preparation subsystem

\section{Visual Servo System Overview}

As shown in Fig. 4, vision servo system for water washing robot consists of image acquisition subsystem, image processing subsystem and robot control subsystem. The image acquisition subsystem consists of binocular stereo camera and infrared laser, the infrared laser will create a laser mark on equipment which will be washed, the binocular stereo camera gets the right and left camera image at the same time through binocular stereo camera. The image processing subsystem receives the images acquired from the binocular camera, and gets the 3-Dimensions information of laser mark on the power equipment through image processing algorithm. The robot control subsystem transforms the spatial coordinate information to control signal which can be accepted by the robot, and plans motion path of the robot. The three subsystems make up a closed-loop system, as shown in Fig.4.

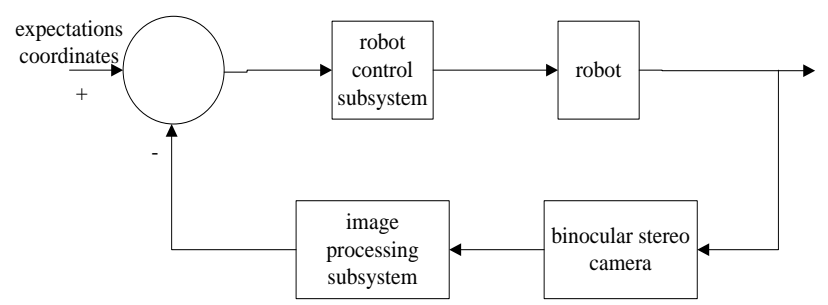

Fig. 4 Block diagram of Vision servo system

The image processing subsystem is the most important part of this system. In stereo vision field, the general method for getting 3-Dimensions information of the working scene is to analyze the left and the right image which got from the binocular camera through image processing algorithm, which will be described in the remaining part of the paper. 


\section{Binocular Stereo Vision Algorithms}

We introduce binocular stereo vision algorithm firstly. The binocular stereo vision algorithm can be divided into three steps[2]. Firstly, the binocular camera must be calibrated not only to correct the camera distortion but also to make sure the polar line in the same line. Secondly, find the corresponding points which are the different projection of the same point in real world on the left and right camera (As shown in 5). The disparity value indicates the distance between the pixels coordinates of the reference pixel and its corresponding position in the other image. For stereo cameras that have their camera coordinate systems aligned, the disparity value is simply the difference in the column positions of the corresponding pixels.

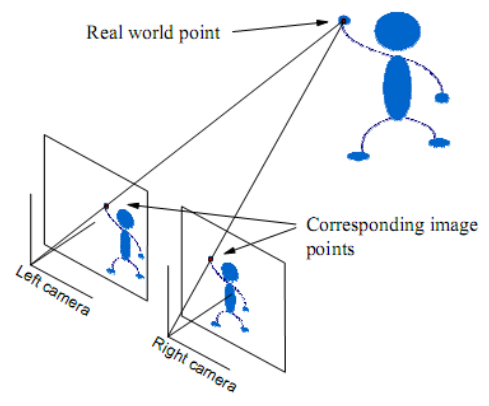

Fig. 5 Binocular stereo vision schematic

Thirdly, calculate the disparity image which describes the column positions deviation of the corresponding points, and calculate the three-dimension information of the images. The equations that given the relationship between $3 \mathrm{D}$ and row, column, disparity is also simple:

$$
Z=\frac{B f}{d} ; \frac{X}{Z}=\frac{u}{f} ; \frac{Y}{Z}=\frac{v}{f}
$$

where $(X, Y, Z)$ is the position of the $3 D$ point in the reference camera coordinate system, $f$ is the camera focal length, $(u, v)$ is the position of the pixel in the image, $d$ is the disparity value and $B$ is the baseline between the cameras. One can see from these equations that the $(X, Y, Z)$ values can be extracted given that the $(u, v)$ and $d$ values are known. $B$ and $f$ are determined by camera calibration and remain constant.

\section{Experiment and Application Situation}

In order to verify the algorithm validity, we apply this system in water washing robot, and analyze the change of distance between the idea goal of the wash position (the infrared laser mark position) and real wash position which is driven by the algorithms mentioned above. As shown in Fig.6, the distances become to zero in 6 seconds, which show that the algorithm has better convergence, and shows the vision system is proved to be validity.

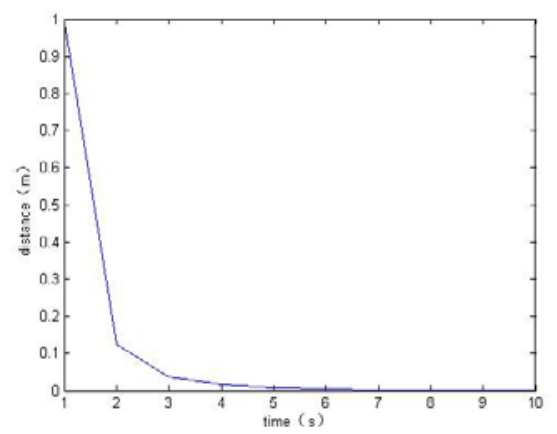

Fig. 6 Curve of distance between robot tool and device

The visual servo system for water washing robot proposed in this paper, has successfully passed the application test in $220 \mathrm{KV}$ laboratory substation, which indicates that the system can meet the 
demand for control of the water washing robot. It can further improve the system's intelligent level and autonomy by using visual servo technology. With the improvement of the system's performance, it's believed that this system will have a great large implement space

\section{References}

[1] Shouyin Lu, Peisun Ma and Hui Qi. Research on high voltage electric power live line working robot. Automation of Electric Power Systems, 2003(17).

[2] Xiangdong Li,Shouyin Lu, Hong Wang. Introduction of the Substation Intelligent Mobile Robot. ShanDong Electric Power, 2005(03).

[3] S.Birchfield and C. Tomasi. Depth discontinuities by pixel to pixel stereo. In Proceedings of the 6th IEEE International Conference on Computer Vision (ICCV ’98), p.1073-1080, 1998.

[4] C.M.Godzhiev. Testing the covariance matrix of a renovation sequence under operating of the Kalman filter. Automation and Remote Control, 1996, 57(7):1046- 1052.

[5] M.Nakashima, K.Yano and Y.Maruyama. The Hot Line Work Robot System "Phase II" and Its Human-robot Interface"Mos". Proc of the IEEE/RSJ International Conference on Intelligent Robots and Systems, 1995, 2:116-123.

[6] A.Santamaria, R.Aracil and A.Tuduri. Teleoperated Robots for Live Power Lines Maintenance (ROBTET) . In: Proc of 14th International Conference and Exhibition on Electricity Distribution, 1997, 3(31):1-5. 